\title{
STUDY OF PERSON IDENTIFICATION USING PALMPRINT RECOGNITION SYSTEM BASED ON MINUTIAE CYLINDRICAL CODE
}

\author{
Priya V. Dudhanale ${ }^{1}$, S.R.Ganorkar ${ }^{2}$ \\ ${ }^{1}$ Student, Dept. of E\&TC, Sinhgad College of Engineering, Pune, India \\ ${ }^{2}$ Associate professor, Dept. of E\&TC, Sinhgad College of Engineering, Pune, India
}

\begin{abstract}
In today's world, biometrics system is used everywhere for the security and personal identification. Palmprint is a very important type of biometrics and it is used mostly in forensic applications. In this paper, high resolution palmprint images are used, feature extraction and matching steps are developed such as system is simple, more accurate and faster. Minutiae Cylindrical code is used for matching purpose. Palmprint recognition is difficult task because of size of large images. For high security applications high resolution palmprints are needed from which more extra information can be extracted. This Palmprint recognition system has many steps to common with fingerprint having optimized and specified steps to process large sized palmprint images. Feature extraction method is used to detect minutiae followed by local matching algorithm.
\end{abstract}

Keywords - Gabor filter,Minutia Cylinder Code, minutiae, palmprint, $x$-signature.

\section{INTRODUCTION}

Today, in our daily life, we are often being asked for verification of our identity. In the modern world there is more necessity to identify individuals and security. For accessing secure there is need to possess password, pin etc. Cybercrime is increased. So biometrics systems are widely used nowadays.

Palmprint is nothing but the inner surface of the hand. Palmprint recognition has an importance in a personal identification technique. Generally palmprint recognition is based on two types low resolution and high resolution of images. Depending on resolutions applications are different. The pattern of ridge is unique for an individual [5]. Palmprint recognition system has been proposed here based on high resolution such as 500dpi which contains Principle lines, ridges, wrinkles and minutiae points. Principle line is heart line, ridges and wrinkles are smaller lines other than principle lines. Minutiae points is nothing but the small details which is mostly used for fingerprint recognition. Plamprint recognition is used because of it is having larger surface area, it is less susceptible to wound and noise and having more features. Typically any biometric system is designed by taking in account some things which are cost, speed, security, accuracy, user acceptance. No two persons have same palmprint. Palm regions, ridges, wrinkles and minutiae point are shown in the following image. Biometric System takes account of following things cost, user acceptance, computation speed, security, accuracy, environment constraints. The following figure shows Palmprint image.

The rest of this paper organized as follows : Section II explains Flowchart and feature extraction methods, Section
III gives Matching methods, Section IV shows Results and finally offers Section V Conclusion.

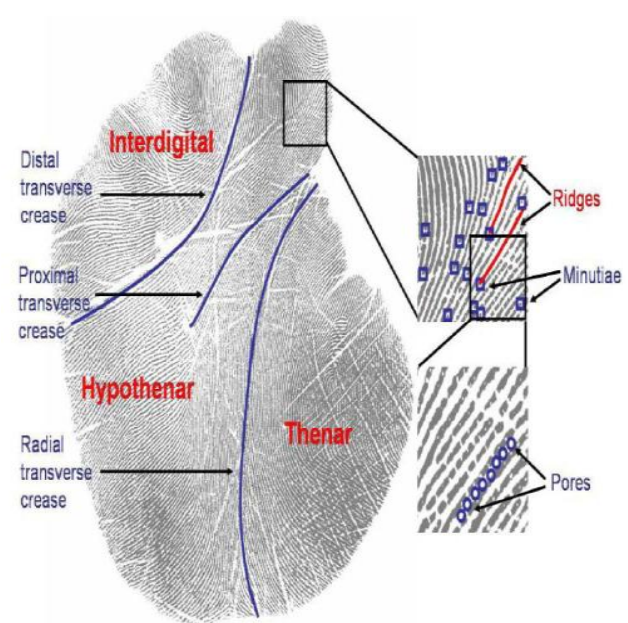

Fig1: The principal lines, regions, ridges, and minutiae in a palmprint [5].

\section{FLOWCHART AND FEATURE EXTRACTION}

Palmprint recognition system having following steps first is the palmprint aquisation where palmprint image is taken from the database, second is preprocessing where palmprint image is segmented as foreground and background with the help of gradient method and image is binarized, by Gaussian and median filtering noise is reduced in this step[11], third step after preprocessing is feature extraction where the features are extracted from pre-processed palmprint images by using gabor filter, final step is the matcher where the palmprint features are compared from training and test data 
sets. If palmprints are matching then it is accepted otherwise it is rejected.

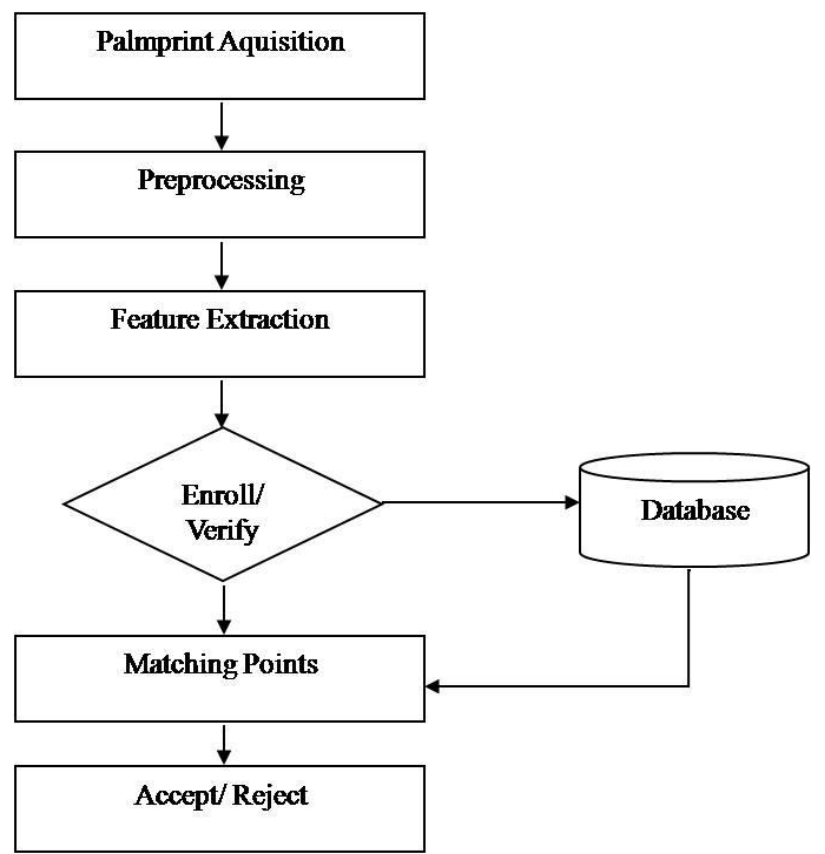

Fig2: Flowchart of Palmprint Recognition Algorithm [3]

For extracting the minutiae points from palmprint images the pre-processed image is enhanced using gabor filter.

Extracted minutiae points should represented as follows: $M$ $=\left\{m \mid m=\left(x_{m}, y_{m}, \theta_{m}\right)\right\}$, where $x_{m}$ and $y_{m}$ represent its location, and $\theta_{m}$ is its direction (in the range $[0,2 \pi[$ ). Minutiae points are matched with the help of Minutiae cylindrical code which is having fixed radius based approach. Therefore it supports to false and missing minutiae points. Border difficulties are handled easily by minutiae cylindrical code by viewing minutiae points which are not near to border as matchable and the points which are near to border as should be matchable.

For matching cylinders system uses bit based coding. In which AND and OR bitwise operations are used. Therefore matching of cylinders is easy and quick.

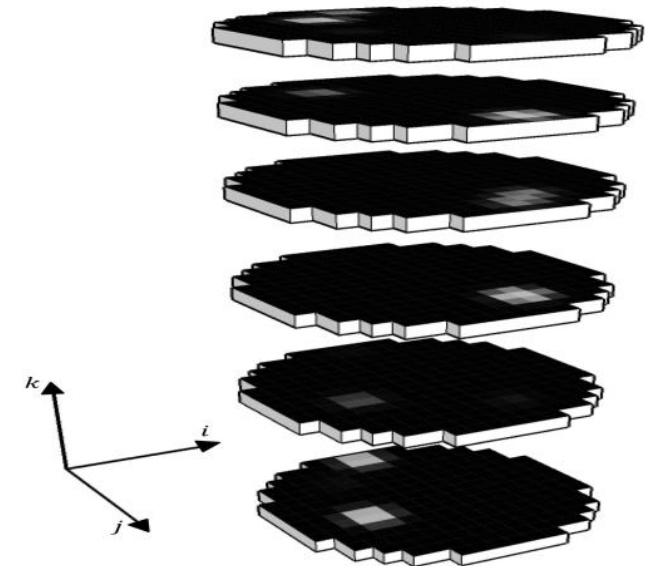

Fig3: A graphical representation of a cylinder [9]
Stochastic Gradient Descent Algorithm : In this algorithm learning system is updated on the basis of loss function. Loss function is a measuring function that how learning system is performing. An average of all loss function gives the cost function which measures over all training examples. Stochastic gradient descent method is more faster than the gradient descent because it calculates gradient of only one example rather than whole training set.[16]

Principle Component Analysis : PCA is sub space approach which is also called appearance based approach and it is used with Euclidean distance classifier to give better results. In this method eigenvectors and eigenvalues are calculated which provides useful information of training data set. This method is very much useful for reducing the dimension of data. Eigenpalms are principle components of palmprint which generated from basis vectors from palmprint images. Eigenvectors are represented variance having large eigenvalues. [15]

This algorithm contains following steps:

[1]. Set of palmprint images like $i_{1}, i_{2}, \ldots \ldots i_{M}$. Calceulate the average palm of set.

[2]. Substract the average from each of the palmprint.

[3]. Calculate covariance matrix.

$$
\text { a. } \mathrm{C}=\sum_{j=1}^{M} \phi_{j} \phi_{j}^{T}
$$

[4]. Calculate eigenvector and eigenvalues of covariance matrix.

[5]. Choose the feature vector.

[6]. Derive new palm images.

\section{MATCHING}

Minutiae cylindrical code is created having radius $\mathrm{R}$ and height $2 \pi$ whose base is kept on minutiae location(x, y). In local matching step, similarity between minutiae points in two cylinders i.e. training and test data set is taken by calculating hamming distance between minutiae points and finally lowest hamming distance between minutiae points are more similar. That means in local matching step there is similarity between local structure is considered. For calculating global score, overall similarity between has to be taken from local similarities.

Hamming distance: Matching algorithm gives the similarity measurement between palmprint images. It is used for binary feature vectors. Hamming distance score is calculated which is in between 1 and 0 . If it is 0 then it is said to be perfectly matched. But it is impractical so threshold value of hamming distance is calculated and lesser the value than threshold value then these two palmprint images are said to be matches.

Let $\mathrm{P}$ and $\mathrm{Q}$ two palmprint matrices of features. Then hamming distance is

$$
D_{0}=\frac{\sum_{i=1}^{N} \sum_{j=1}^{N} P_{R}(\mathrm{i}, \mathrm{j}) \otimes Q_{R}(\mathrm{i}, \mathrm{j})+P_{I}(\mathrm{i}, \mathrm{j}) \hat{\otimes} Q_{I}(\mathrm{i}, \mathrm{j})}{2 N^{2}}
$$


Where $P_{R}, Q_{R}, P_{I}, Q_{I}$ are the real and imaginary parts of $\mathrm{P}(\mathrm{Q})$.

Euclidean Distance : Euclidean distance computes the summation of squared differences between two feature vectors. It falls in the range $[0,1]$, it measures the distance between palmprint images and gives matching result i.e. similar or dissimilar. If it is similar lower the Euclidean distance, it should be zero. Formula of Euclidean distance is as follows:

$$
\text { Distance }=\sqrt{\sum_{l=1}^{k}\left(F V_{i, l}-F V_{j, l}\right)^{2}}
$$

$F V_{i, l}$ it is a feature vector $\mathrm{i}$ with length $\mathrm{k}$.

\section{RESULTS}

Nowadays high resolution palmprint database available is only Tsinghua Palmprint Database (THUPALMLAB) which contains 1280 palmprint images having resolution 500dpi and image size is $2040 \times 2040$ pixels.

To judge the presented system, protocol used is as follows:

First is calculate false nonmatch rate by equating each impression against the rest ones of the same palm.

Second is calculate false match rate by equating each impression of each palm against each impression of rest palms in test set.

Cylinder radius, Number of cells along the cylinder diameter, Number of cylinder sections, Maximum global rotation allowed between two palmprints, Sigmoid parameters, Weight parameter are main parameters of the matching algorithm. Note that parameter $\partial_{\theta}$ has been set to $\pi$, since in the THUPALMLAB database any rotation angle between two palmprints is possible.

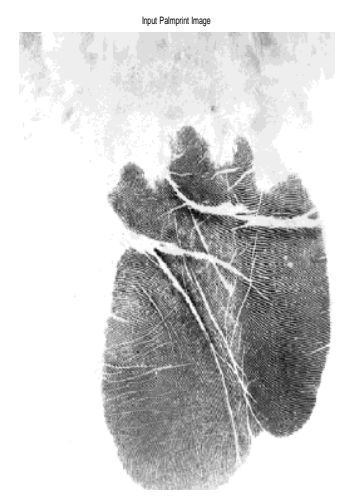

Fig4: Input Palmprint Image
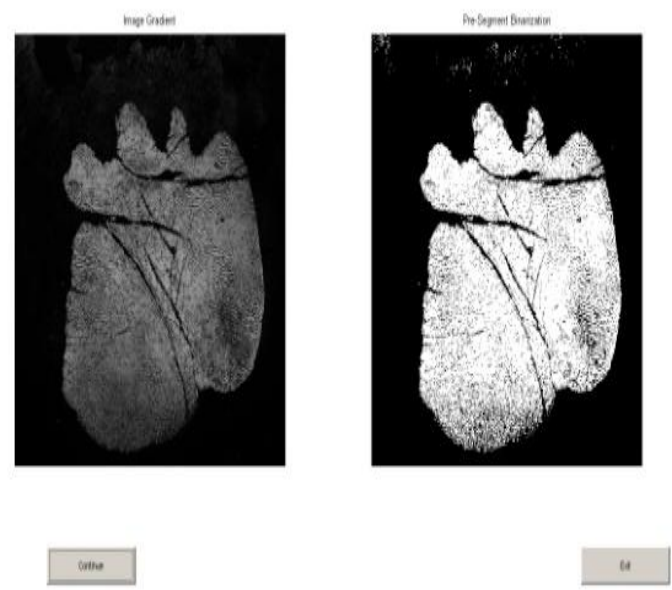

Fig5: Image Gradient and Pre-Segment Binarization
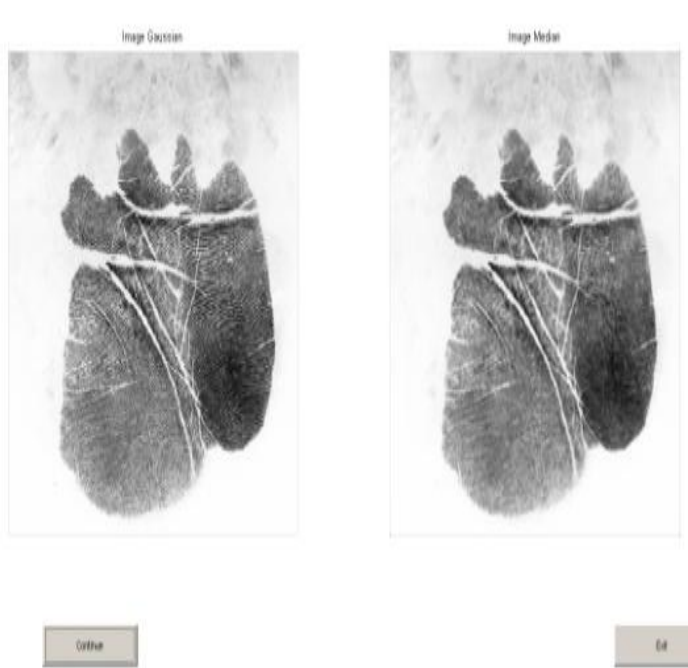

Fig6: Image Gaussian and Image Median
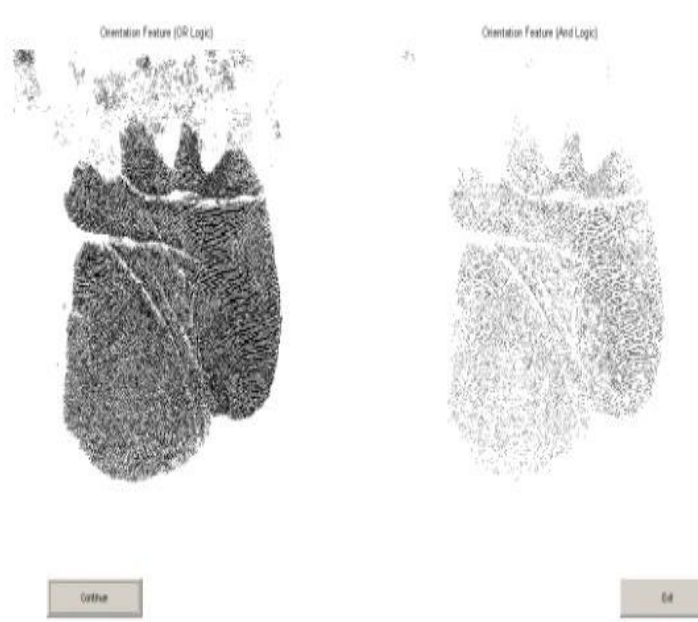

Fig7: Orientation Feature (OR Logic) and Orientation Feature (AND Logic) 


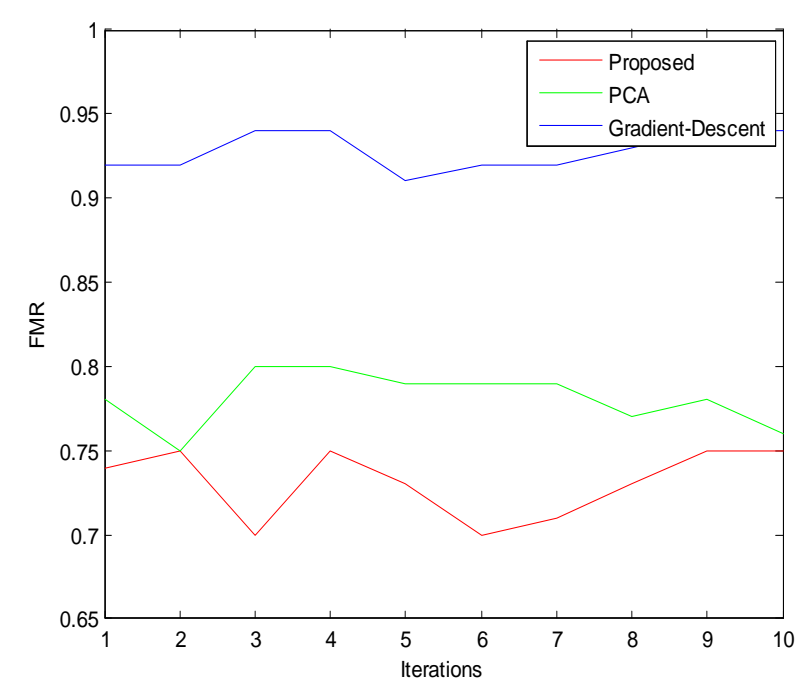

Fig 8 : FMR Graph of Proposed method, PCA, Gradient Descent

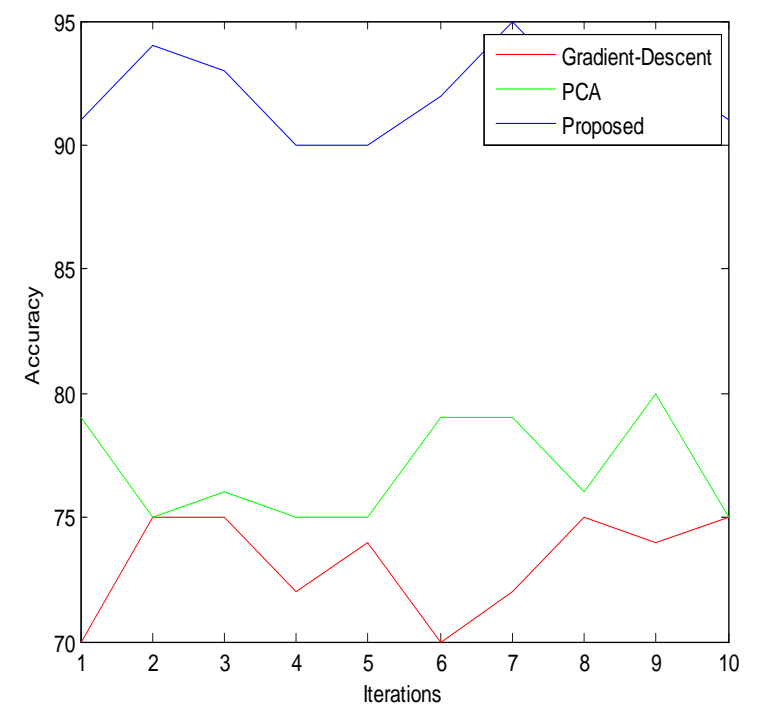

Fig9: Accuracy Graph of Proposed method, PCA, Gradient Descent

\section{CONCLUSIONS}

Palmprint is most successful biometric trait that mostly used today.In this paper a high resolution palmprint recognition system is introduced. In which features i.e. minutiae points are extracted by using Gabor filter. In matching method i.e. Minutiae cylindrical code is robust against skin distortion having fixed radius and reduces the border problems using hamming distance algorithm. Feature extraction and matching methods are designed such as complexity is low, fast and accurate.

Feature extracted using Gabor filter than Stochastic Gradient Descent and Principle Component Analysis are more accurate. So, here we proposed that Gabor filters with Hamming distance is best method for Palmprint Recognition.

\section{REFERENCES}

[1]. A. Jain, P. Flynn, and A. Ross, Handbook of Biometrics. New York:Springer-Verlag, 2007.

[2]. -D. Maltoni, D. Maio, A. K. Jain, and S. Prabhakar, Handbook of Fingerprint Recognition, 2nd ed. New York: Springer-Verlag, 2009.

[3]. -A. Kong, D. Zhang, and M. Kamel, "A survey of palmprint recognition," Pattern Recognit., vol. 42, no. 7, pp. 1408-1418, Jul. 2009.

[4]. A. K. Jain and J. Feng, "Latent palmprint matching," IEEE Trans. Pattern Anal. Mach. Intell., vol. 31, no. 6, pp. 1032-1047, Jun. 2009.

[5]. J. Dai and J. Zhou, "Multifeature based high resolution palmprint recognition," IEEE Trans. Pattern Anal. Mach. Intell., vol. 33, no. 5, pp. 945-957, May 2011.

[6]. G. Lu, K. Wang, and D. Zhang, "Wavelet based feature extraction for palmprint," in Proc. 2nd Int. Conf. Image Graph., 2002, pp. 780-784.

[7]. L. Zhang and D. Zhang, "Characterization of palmprints by wavelet signatures via directional context modeling," IEEE Trans. Syst., Man, Cybern. B, Cybern., vol. 34, no. 3, pp. 1335-1347, Jun. 2004.

[8]. R. Cappelli, M. Ferrara, and D. Maltoni, "Minutia cylinder-code: A new representation and matching technique for fingerprint recognition," IEEE Trans. Pattern Anal. Mach. Intell., vol. 32, no. 12, pp. 2128-2141, Dec. 2010.

[9]. R. Cappelli, M. Ferrara, and D. Maltoni, "Fingerprint indexing based on minutia cylinder-code," IEEE Trans. Pattern Anal. Mach. Intell., vol. 33, no. 5, pp. 1051-1057, May 2011.

[10]. D. Maio and D. Maltoni, "Direct gray-scale minutiae detection in fingerprints," IEEE Trans. Pattern Anal. Mach. Intell., vol. 19, no. 1, pp. 27-40, Jan. 1997.

[11]. F. Turroni, D. Maltoni, R. Cappelli, and D. Maio, "Improving fingerprint orientation extraction," IEEE Trans. Inf. Forensics Security, vol. 6, no. 3, pp. 1002-1013, Sep. 2011.

[12]. L. Hong, Y. Wan, and A. K. Jain, "Fingerprint image enhancement: Algorithms and performance evaluation," IEEE Trans. Pattern Anal. Mach. Intell., vol. 20, no. 8, pp. 777-789, Aug. 1998.

[13]. Tsinghua Univ. Dept. Autom., i-Vision Group Web Site, 2011. [Online]. Available: http://ivg.au.tsinghua.edu.cn [14]. R. Cappelli, M. Ferrara, and D. Maio, "A Fast and Accurate Palmprint Recognition System Based on Minutiae," IEEE Trans. Systems, Man, and Cybernetics, Part B: Cybernetics, vol. 42, no. 3,pp. 956-962, June 2012. [15]. Tee Connie, Andrew Teoh, Michael Goh, David Ngo "Palmprint Recognition with PCA and ICA", in Proc. 2nd Int. Conf. Image Graph., 2008.

[16]. Available online http://leon.bottou.org 